\title{
Enhancement of active shuttle avoidance response by the NO-cGMP-PKG activator YC-1
}

\author{
Wei-Lin Chien ${ }^{a}$, Keng-Chen Liang ${ }^{b}$, Wen-Mei Fu ${ }^{\text {a,* }}$ \\ a Pharmacological Institute, College of Medicine, National Taiwan University, Taipei, Taiwan \\ b Department of Psychology, National Taiwan University, Taipei, Taiwan
}

\section{A R T I C L E I N F O}

\section{Article history:}

Received 6 February 2008

Received in revised form 23 May 2008

Accepted 8 June 2008

Available online 15 June 2008

\section{Keywords:}

YC-1

BDNF

Amygdala

Fear memory

Active shuttle avoidance

\begin{abstract}
A B S T R A C T
Although much has been learned about the role of the amygdala in Pavlovian fear conditioning, relatively little is known about the signaling pathway involved in the acquisition of an active avoidance reaction. The aim of this study is to investigate the potentiating effects of the NO-guanylate cyclase activator YC- 1 on learning and memory of shuttle avoidance test in rats. YC-1 enhanced the induction of long-term potentiation (LTP) in amygdala through NO-cGMP-PKG-ERK pathway and the increase of BDNF expression. The Western blot and PCR methods were used to examine the signaling pathways involved in fear memory. It was found that YC-1 increased the avoidance responses during learning period and the memory retention lasted longer than one week. The enhancement of learning behavior by $\mathrm{YC}-1$ was antagonized by intracerebroventricular injection of NOS inhibitor L-NAME, PKG inhibitor Rp-8-Br-PET-cGMPS and MEK inhibitor PD98059, indicating that NO-CGMP-PKG and ERK pathways are involved in the learning potentiating action of YC-1. In addition, YC-1 increased the activation of ERK and Akt 30 min after Day-1 training in amygdala. YC-1 also potentiated the expression of BDNF and CREB in response to fear memory test. Taken together, these findings suggest that NO-cGMP-PKG-ERK signaling pathway is involved in the action of YC-1 in enhancing the fear memory.
\end{abstract}

(C) 2008 Elsevier B.V. All rights reserved.

\section{Introduction}

Learning and memory are the most intensively studied subjects in neuroscience. Various approaches have been used to understand the underlying cellular and molecular mechanisms (Bailey et al., 2004; Kim and Jung, 2006; Wang et al., 2006). In recent years, however, considerable progress has been made in the elucidation of the key circuits and structures that mediate Pavlovian auditory fear conditioning, a behavioural task in which a neutral stimulus (conditioned stimulus) acquires the ability to elicit fear responses following its association with an aversive unconditioned stimulus, such as an electric shock (LeDoux, 2000; Maren, 2005). Converging evidence from a variety of studies points to the amygdala, as an important site of plasticity in fear conditioning (LeDoux, 2000; Schafe et al., 2005b). The alterations of synaptic transmission in amygdala are thought to subserve the encoding of fear memory (Rodrigues et al., 2004; Maren, 2005). In addition to amygdala, several brain regions including hippocampus, cortex and cerebellum are also reported to exert

\footnotetext{
* Corresponding author. Pharmacological Institute, College of Medicine, National Taiwan University, No. 1, Sec. 1, Jen-Ai Road, Taipei, Taiwan. Tel.: +886 2 23123456x8319; fax: +886 223417930

E-mail address: wenmei@ntu.edu.tw (W.-M. Fu).
}

important roles in acquisition, storage and expression of fear memory (Kim and Jung, 2006).

It is reported that $\mathrm{NO}$ is produced in the amygdala during acquisition of the conditioning fear memory (Sato et al., 2006). Although much has been learned about Pavlovian fear conditioning, relatively little is known about the role of NO in more complex aversive learning, such as two-way active shuttle avoidance test (Johnson et al., 2000; Schafe et al., 2005a). We previously reported that a novel synthetic compound YC-1, 3-(5'-hydroxymethyl-2'furyl)-1-benzylindazole, enhanced LTP in amygdala and hippocampus in a concentraction-dependent manner and greatly improved rodent learning behavior when examined with the Morris water maze and passive avoidance tests (Chien et al., 2003, 2005). Here we further investigated the signaling pathway involved in the LTP enhancement of YC-1 in amygdala as well as fear memory potentiation in active shuttle avoidance test.

Since extracellular signal-regulated kinase (ERK) is found in several brain regions associated with learning and memory, numerous studies are now focusing on ERK in regulating neuronal function and memory process (Sweatt, 2004; Herry et al., 2006). ERK activation is required for the establishment of long-term potentiation (LTP) in lateral amygdala and for the acquisition, consolidation and extinction of fear conditioning (Rodrigues et al., 2004; Shalin et al., 2004; Herry et al., 2006). Phosphatidylinositol 3-kinase (PI3 K) has been shown to 
interact with ERK and inhibition of PI3 K may affect phosphorylation of Akt and member proteins of ERK pathway in synaptic plasticity and memory processes (Lin et al., 2001; Opazo et al., 2003; Chen et al., 2005).

Brain-derived neurotrophic factor (BDNF), a member of the neurotrophin family, plays an important role in neuronal growth and development and also in synaptic structure and function in adult nervous system (Poo, 2001; Soule et al., 2006). It is also found that BDNF levels are positively correlated with the ability to learn a spatial task (Alonso et al., 2005; Francia et al., 2006) as well as the acquisition and consolidation of fear memory (Chhatwal et al., 2006; Ou and Gean, 2006). The mRNA of BDNF but not other neurotrophic factors significantly increases in basolateral amygdala following fear conditioning (Rattiner et al., 2005). We previously found that YC-1 enhanced the phosphorylation of ERK and CREB (Chien et al., 2003). It is also reported that the phosphorylated CREB binds to BDNF promoter and up-regulates the expression of BDNF in amygdala, which helps the consolidation of fear memory (Ou and Gean, 2007). We here also investigated the role of BDNF in the fear memory enhancement by YC-1.

\section{Materials and methods}

\subsection{Animal}

All protocols complied with institutional guidelines and were approved by Animal Care Committees of Medical College, National Taiwan University. Wistar rats were used for electrophysiological recording (150-250 g) and active shuttle avoidance test (200-300 g). Wistar rats used for studies were housed in pairs and housed individually after surgery. Animals were housed and maintained on a 12-h-on/12-h-off light/dark cycle. All animals were allowed free access to food and water.

\subsection{Materials}

$N^{\mathrm{G}}$-nitro-L-arginine methyl ester (L-NAME), $1 \mathrm{H}$-[1,2,4] oxadiazolo [4,3-a] quinoxalin-1-one (ODQ), $\beta$-Phenyl-1, $N^{2}$-etheno-8-bromoguanosine-3',5'-cyclic mnophosphorothioate, Rp-isomer (Rp-8-Br-PETcGMPS), 2'-amino-3'-methoxyflavone (PD98059), K252a and picrotoxin were purchased from Sigma-Aldrich (St. Louis MO). Avidin-biotinperoxidase detection kit was purchased from Vector Laboratories (Vectastain ABC kit; Vector Laboratories, Burlingame, CA). 3-(5'hydroxymethyl-2'-furyl)-1-benzylindazole (YC-1) was provided by Yung-Shin Pharmaceutical Industry Co. Ltd. (Taichung, Taiwan). The Primary antibodies used for Western blot were purchased from Santa Cruz Biotechnology (Santa Cruz biotechnology, California, USA).

\subsection{Electrophysiological recordings in amygdala}

Male Wistar rats were sacrificed by cervical dislocation, and brains were quickly removed and placed in cold dissection buffer with the following composition: $87 \mathrm{mM} \mathrm{NaCl}, 2.5 \mathrm{mM} \mathrm{KCl}, 1.26 \mathrm{mM} \mathrm{NaH}_{2} \mathrm{PO}_{4}$, $25 \mathrm{mM} \mathrm{NaHCO}_{3}, 0.5 \mathrm{mM} \mathrm{CaCl}_{2}, 5.0 \mathrm{mM} \mathrm{MgSO}_{4}, 25 \mathrm{mM}$ glucose, $75 \mathrm{mM}$ sucrose, saturated with $95 \% \mathrm{O}_{2}$ and $5 \% \mathrm{CO}_{2}$. Coronal slices $(400 \mu \mathrm{m})$ containing amygdala were cut with a vibroslicer in cold $\mathrm{O}_{2} / \mathrm{CO}_{2}$ bubbled dissection buffer. Slices were then maintained in an interface chamber at room temperature for at least $1.5 \mathrm{~h}$ before recording, where they were subfused with aCSF consisting of $125 \mathrm{mM} \mathrm{NaCl}$, $2.5 \mathrm{mM} \mathrm{KCl}, 1.26 \mathrm{mM} \mathrm{NaH}_{2} \mathrm{PO}_{4}, 25 \mathrm{mM} \mathrm{NaHCO}_{3}, 2.0 \mathrm{mM} \mathrm{CaCl}_{2}, 1.0 \mathrm{mM}$ $\mathrm{MgSO}_{4}, 25 \mathrm{mM}$ glucose, bubbled with $95 \% \mathrm{O}_{2}$ and $5 \% \mathrm{CO}_{2}$. A bipolar tungsten-stimulating electrode was placed close to the medial border of lateral nucleus of amygdala (thalamic pathway), and extracellular field potentials were recorded using a glass microelectrode (3 M $\Omega$, filled with aCSF), placed in the lateral amygdala. The pulse duration was $100 \mu$ s, and test responses were elicited at $0.05 \mathrm{~Hz}$. The perfusion rate of aCSF was $2 \mathrm{ml} / \mathrm{min}$. Baseline responses were monitored for 10 min prior to tetanus. LTP was induced with tetanized stimulation ( $100 \mathrm{~Hz}$ for $1 \mathrm{~s}$, two times at $20 \mathrm{~s}$ interval) and the stimulation strength was set to provide fEPSP with an amplitude of 40-60\% to maximum. The stimulation intensity for LTP induction in each slice was the same as that used to elicit baseline EPSPs. Picrotoxin (10 $\mu \mathrm{M}$; Sigma) was included in the bath in all experiments to block GABAergic inhibitory effects. The initial slope of the field EPSP was measured and LTP at each time point was expressed as a percentage of baseline. The concentration of YC-1 was chosen according to our previous experiment (Chien et al., 2003) and the concentrations of antagonists were tested by pilot studies for choosing the minimum concentrations which can inhibit the enhancement of YC-1 but left the control LTP induction unaffected.

\subsection{Surgical procedure}

The cannula was positioned under anaesthesia $(200 \mathrm{mg} / \mathrm{kg}$ trichloroacetaldehyde) at $1.0 \mathrm{~mm}$ lateral to the midline, $1.5 \mathrm{~mm}$ caudal to bregma, and $3.5 \mathrm{~mm}$ below the surface of the skull, according to the atlas of Paxinos \& Watson (1997). Cannula was made of 23-guage stainless steel tubing, with a $0.33-\mathrm{mm}$ inner diameter, a $0.63-\mathrm{mm}$ outer diameter and a length of $10 \mathrm{~mm}$. Dental cement was used to secure the cannula to two screws inserted in the skull. Rats were kept warm until recovery from anaesthesia. They recuperated for at least 1 week before being subjected to behavioural testing. Rats were housed individually after surgery and kept under these conditions until the end of the experiment.

\subsection{Two-way active avoidance test}

Active avoidance test was conducted in $45 \times 22 \times 33 \mathrm{~cm}$ two-way automated shuttle-box (ShuttleFlex Active/Passive avoidance system, AccuScan Instruments, Inc.). The compartment floor was electrifiable and made of stainless steel bars. The shuttle-box was divided into two equal compartments by gray plank with a square hole $(8 \times 10 \mathrm{~cm})$ in the center of plank, allowing a communication between two experimental chambers. The shuttle-box was situated in the dark and quiet room. The conditioned stimulus was light, emitted by two $2.8 \mathrm{~W}$ light bulbs, and $85 \mathrm{~dB}$ tone of $3 \mathrm{~s}$ duration. The unconditioned stimulus was a foot shock $(2 \mathrm{~s}, 0.8 \mathrm{~mA})$ delivered to the grid floor. In trials, the conditioned stimulus was followed by the unconditioned stimulus. The interval between the conditioned and unconditioned stimulus was a short delay conditioning procedure, in which the unconditioned stimulus was presented immediately after the end of the conditioned stimulus. The trials were given with a $25 \mathrm{~s}$ intertribal interval (Carageorgiou et al., 2003). The shuttle-box was connected to a computer that controlled the training schedule and scored avoidance, escape and non-responses, latency and the number of crossing.

Active avoidance was compared among three groups: (1) shamgroup (2) 0.1\% DMSO-injected control group (3) YC-1-injected rats. YC-1 $(1 \mathrm{mg} / \mathrm{kg})$ or vehicle $(0.1 \%$ DMSO for the sham and control groups) was injected (i.p.) $10 \mathrm{~min}$ before the first trial. Vehicle, NOS inhibitor L-NAME (1 $\mu \mathrm{mol})$, PKG inhibitors Rp-8-Br-PET-cGMPS (1 nmol) or MEK inhibitor PD98059 (1 nmol) was slowly administered by intracerebroventricular (i.c.v., $1 \mu \mathrm{L} / \mathrm{min}$ ) injection 20 min before the first trial. The doses of drugs were chosen according to our previous study (Chien et al., 2005). On the first training day, rats were placed in the apparatus for $10 \mathrm{~min}$ to explore the apparatus and to be familiar with the learning environment (Torras-Garcia et al., 2003). The exploration time was reduced to $90 \mathrm{~s}$ between the $2 \mathrm{nd}$ and 4 th day. A conditioned response was recorded as if the rat avoided the shock by running from one compartment to the other one during the conditioned stimulus. The sham-rats received the same manipulation as the controls but did not have the unconditioned stimulus (foot- 
shock punishment). Besides the number of avoidance responses, intertribal crossing and crossings during the free ambulation period were also scored. One week after training, rats received another test for the evaluation of the long-term memory. The performance of each rat was automatically recorded and the learning ability was assessed as the number of avoidance response. The higher number of avoidance response represents the better learning ability.

\subsection{Western blot analysis}

Rats received two-way active shuttle avoidance test were sacrificed by decapitation at a specific time as mentioned in the results section. The brain areas of amygdala, hippocampus, cortex and cerebellum were sonicated briefly in ice-cold RIPA buffer ( $50 \mathrm{mM}$ Tris$\mathrm{HCl}$, pH 7.5, $0.3 \mathrm{M}$ sucrose, 5 mM EDTA, 2 mM sodium pyrophosphate, $1 \mathrm{mM}$ sodium orthovanadate, $1 \mathrm{mM}$ PMSF, $10 \mu \mathrm{g} / \mathrm{ml}$ leupeptin, and $10 \mu \mathrm{g} / \mathrm{ml}$ aprotinin). After sonication, samples were centrifuged at $14,000 \mathrm{rpm}$ for $30 \mathrm{~min}$, and the supernatant was obtained after pelleting the crude membrane fraction. Protein expression levels of $\mathrm{p}$ ERK, p-Akt, c-Fos and BDNF were determined by Western blotting. Protein concentration in the soluble fraction was measured using a BCA protein assay kit (Pierce), with BSA as the standard. Equivalent amounts of protein for each sample were resolved in $12 \%$ SDSpolyacrylamide gels, blotted electrophoretically to Immobilon and blocked overnight in TBS buffer (50 mM Tris-HCl, pH 7.5, and $150 \mathrm{mM}$ $\mathrm{NaCl}$ ) containing $10 \% \mathrm{BSA}$. For detection of the protein level, blots were incubated with mouse anti-phosphorylated-ERK (1:1000), rabbit antiphosphorylated-Akt (1:1000), ERK (1:2000), c-Fos (1:2000) and BDNF $(1: 1000)$ primary antibodies diluted in TBS. Mouse anti- $\alpha$-tubulin $(1: 2,000)$ was used to evaluate the loading protein. Blots were then incubated at room temperature for $60 \mathrm{~min}$, with an HRP-conjugated secondary antibody (1:2000, Amersham Life Sciences). Protein bands were detected using the ECL kit (NEN Life Science Products, Boston, MA). The density of immunoblots was determined using an image analysis software ImageQuant.

\subsection{Immunocytochemistry}

Rats were anaesthetized and sacrificed $2 \mathrm{~h}$ after training on Day- 2 . The brain sections were washed three times in PBS ( $\mathrm{pH} 7.4$ ), incubated 10 min in $0.3 \% \mathrm{H}_{2} \mathrm{O}_{2}$ and $10 \%$ methanol in PBS, washed twice in PBS, and then incubated with $10 \%$ non-fat milk and $1 \%$ Triton-X 100 in PBS. The brain slices were washed three times in PBS, then incubated with rabbit anti-c-Fos (1:1000) or anti-BDNF (1:200; Santa Cruz) at $4 \mathrm{C}$ for $24 \mathrm{~h}$ in the presence of $4 \%$ normal goat serum. The slices were then incubated with goat anti-rabbit secondary antibody (1:1000; Vector Laboratories) in the presence of $0.1 \%$ triton X-100 for $1 \mathrm{~h}$ and then avidin-biotin-peroxidase complex (Vector Laboratories) for $1 \mathrm{~h}$. The labeling was then revealed by treatment with $0.01 \%$ hydrogen peroxide and 0.05\% 3,3'-diaminobenzidine (DAB, Sigma). Labeled tissue sections were then mounted on gelatin-coated slides and analyzed under a microscope.

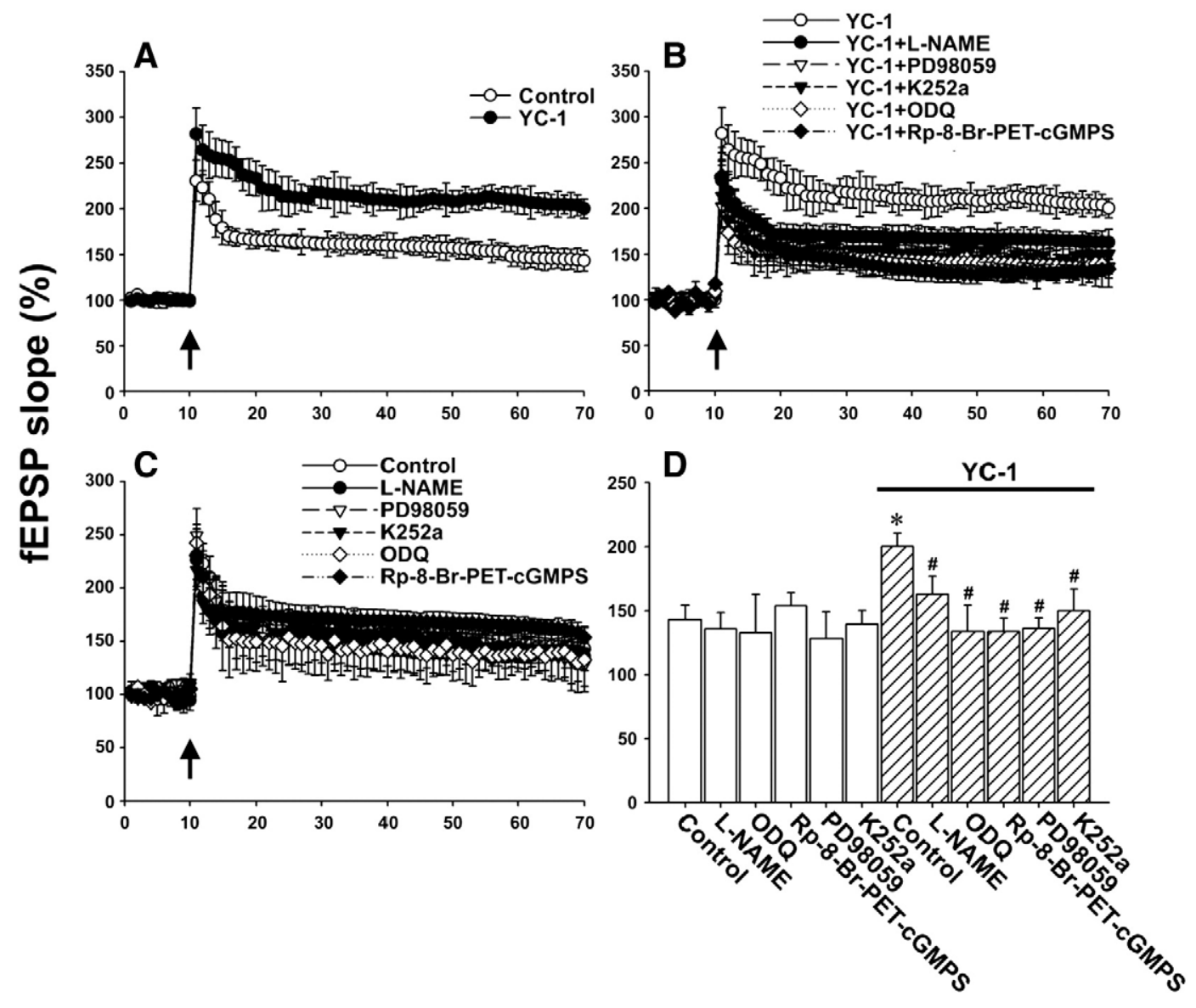

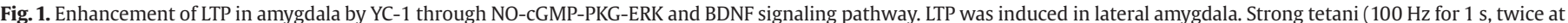

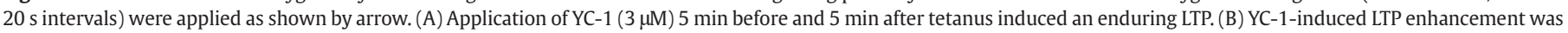

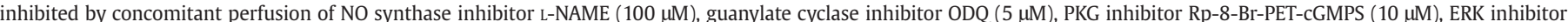

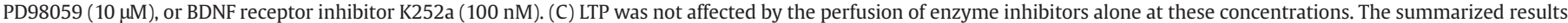

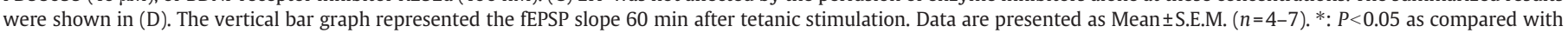
control without YC-1. \#: $P<0.05$ as compared with control in YC-1-treated group. 
2.8. Reverse transcriptase polymerase chain reaction (RT-PCR) for $m R N A$ analysis

Total RNA was extracted by TRIZol Kit (MDBio Inc, Taipei, Taiwan) 30 min after avoidance test on Day-2 and was used for RT-PCR using SuperScriptIII RT-PCR kit (Invitrogen, Carlsbad, CA). Amplification by Tag DNA polymerase (MDBio Inc, Taipei, Taiwan) was accomplished at 28-32 cycles, which was within the linear change of PCR product. In brief, sense and antisense primers were added and the following profile was used: $1 \mathrm{cycle}$ at $94{ }^{\circ} \mathrm{C}$ for $3 \mathrm{~min}$, followed by set cycles at: $94{ }^{\circ} \mathrm{C}$ for $30 \mathrm{~s} ; 55^{\circ} \mathrm{C}$ for $60 \mathrm{~s}$ and $72{ }^{\circ} \mathrm{C}$ for $1 \mathrm{~min}$. All primers used are listed as follows:

\section{GAPDH, sense, GCCATCAACGCCCCTTCATTGAC; antisense, ACGGAAGGCCATGCCAGTGAGCTT; CREB, sense, CTGATTCCCAAAAACGAAGG; antisense, CTGCCCACTGCTAGTTTGGT; BDNF, sense, GACTCTGGAGAGCGTGAAT; antisense, CCACTCGCTAATACTGTCAC;}

PCR products were then separated electrophoretically in a $2 \%$ agarose DNA gel and stained with ethidium bromide. mRNA levels were normalized to the levels of GAPDH.

\subsection{Statistics}

The values given are means \pm S.E.M. For LTP analysis, fEPSP slopes during the last $1 \mathrm{~min}$ of the recording session were compared using a paired two-tailed $t$ test. Active shuttle avoidance tests were analyzed using two-way ANOVA followed by post-hoc Scheffe test. The significance of Western blotting (for p-ERK, p-Akt, c-Fos and BDNF) and PCR (for BDNF and CREB) analysis between the experimental groups and control was assessed by Student's $t$ test. The difference is significant if the $P$ value is less than 0.05 .

\section{Results}

\subsection{YC-1 enhances LTP in amygdala}

NO contributes widely to synaptic plasticity and memory formation (Prast and Philippu, 2001; Schafe et al., 2005a). Here we investigated the effect of YC-1 on LTP signaling at thalamic input synapse to the lateral amygdala by stimulating the fiber through the ventral striatum just medial to the lateral amygdala. As shown in Fig. 1A, perfusion of YC$1(3 \mu \mathrm{M})$ for $10 \mathrm{~min}$ ( $5 \mathrm{~min}$ before and $5 \mathrm{~min}$ after tetanus) significantly increased the extent of LTP. The fEPSP slope was $143.0 \pm 11.1 \%(n=7)$ and $200.0 \pm 10.5 \%(n=7)$ of the control baseline $60 \mathrm{~min}$ after tetanization without or with YC-1 treatment, respectively.

The main effector of NO in many tissues is soluble guanylate cyclase (sGC). Through binding to the heme region of sGC, NO triggers the production of cGMP. We have previously shown that YC-1 enhances LTP in hippocampus through NO-cGMP-PKG-ERK signaling pathway (Chien et al., 2002). Here we also found that concomitant treatment of YC-1 with L-NAME (100 $\mu \mathrm{M}$; Fig. 1B) significantly attenuated the potentiation effect of YC-1, suggesting that NO was involved in the LTP enhancement of YC-1 in amygdala. Concomitant treatment of YC-1 with $1 \mathrm{H}-[1,2,4]-$ oxadiazol(4,3- $\alpha$-)-quinoxalin-1-one (ODQ; $5 \mu \mathrm{M}$ ), Rp-8-Br-PET-cGMPS $(10 \mu \mathrm{M})$ and PD98059 $(10 \mu \mathrm{M})$ also antagonized the enhancement of YC$1(130.1 \pm 25.8 \%, n=5,133.7 \pm 10.2 \%, n=6,136.0 \pm 8.7 \%, n=5$, respectively, Fig. 1B). BDNF plays an important role in regulating the synaptic plasticity of LTP and conditioning fear memory (Rattiner et al., 2005; Ou and Gean, 2007). Treatment of BDNF receptor trkB inhibitor K252a $(100 \mathrm{nM})$ also inhibited the LTP enhancement of YC-1 (149.7 $\pm 17.1 \%$, $n=5$, Fig. 1B). These inhibitors alone at the same concentrations did not affect the induction of LTP (Fig. 1C, $n=4-6$ ). The summarized results are shown in Fig. 1D.

\subsection{YC-1 enhances the avoidance response in shuttle avoidance test}

It is also reported that NO signaling is required for LTP at thalamic inputs to the lateral amygdala and for the long-term consolidation of auditory fear conditioning (Schafe et al., 2005a). We thus investigated the effect of endogenously released NO and YC- 1 on the acquisition of fear memory using active shuttle avoidance test. The rats received 30 training trials each day. YC-1 was administered (i.p., $1 \mathrm{mg} / \mathrm{kg}$ ) $10 \mathrm{~min}$ prior to each daily training session. The mean number of door crossings during the 10 min exploration of the shuttle apparatus prior to avoidance training was the same between control and YC-1-treated group, indicating the exploring activity was not affected by YC-1 (Fig. 2A). As shown in Fig. 2B, avoidance response increased gradually during the 4 days' training period in control rats and YC-1 enhanced the avoidance response rate over the whole training period. Furthermore, even if the test was performed one week after the last

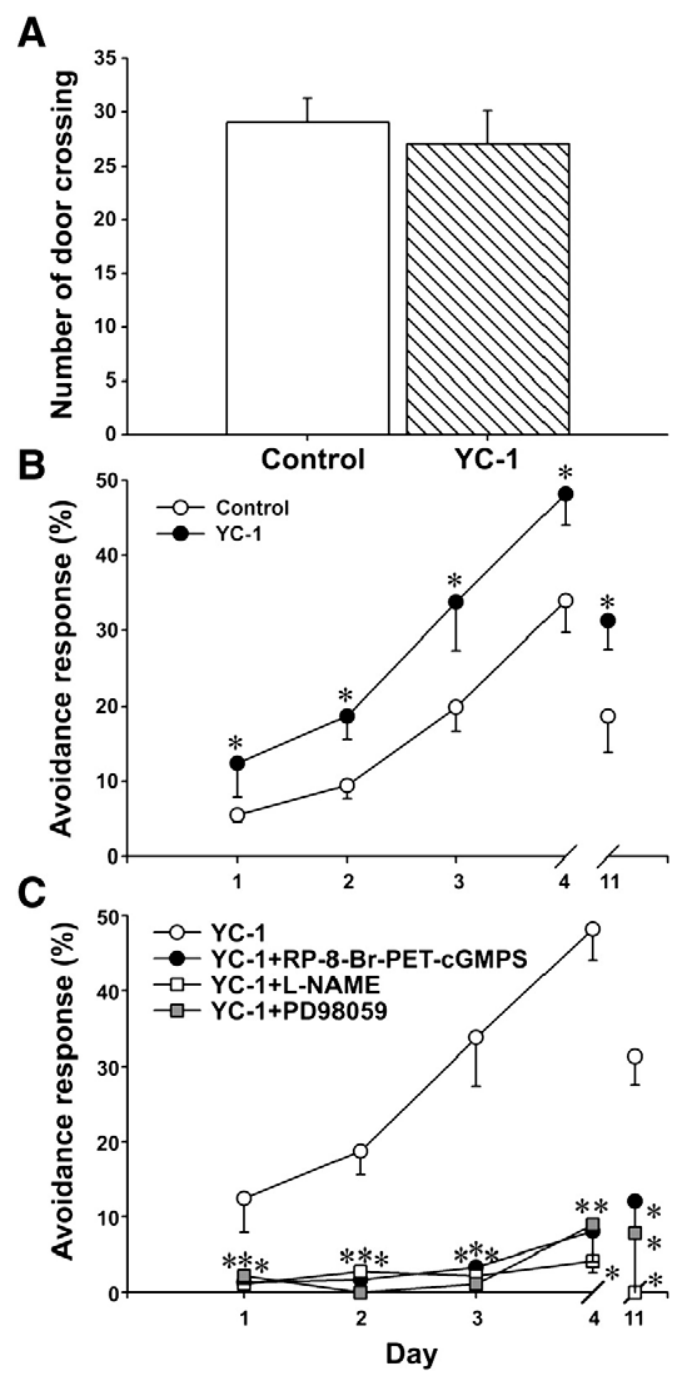

Fig. 2. Enhancement of active shuttle avoidance task by YC-1. (A) On the first training day, the rat was placed in the chamber for $10 \mathrm{~min}$ to explore the apparatus. The number of door crossing between the two compartments was recorded. There was no difference between control ( $n=16)$ and YC-1-treated rats $(n=14)$, indicating that two groups of rats exerted the similar exploring activity. (B) The summarized time-dependent avoidance responses (\%). Note that YC-1 increased the avoidance responses throughout the whole experimental period. Data are presented as Mean \pm S.E.M. ( $n=16$ and 14 for control and YC-1-treated group, respectively). (C) YC-1 (i.p., $1 \mathrm{mg} / \mathrm{kg}$ ) was concomitantly injected with L-NAME (i.c. v., $1 \mu \mathrm{mol}$ ), PD98059 (1 nmol), Rp-8-Br-PET-cGMPS (1 nmol) or saline $10 \mathrm{~min}$ before daily training. Note that L-NAME, PD98059 and Rp-8-Br-PET-cGMPS antagonized the learning potentiating effects of YC-1. Data are presented as Mean \pm S.E.M. $(n=8-14)$. 
day's training; YC-1-treated rats still possessed the higher response rate. The mean avoidance response on Day-11 was $18.7 \pm 4.8 \%(n=16)$ and $33.3 \pm 3.0 \%(n=14)$ for control and YC-1-treated rats, respectively (ANOVA test among two groups: $F_{1,29}=9.435, P<0.001$ ). These results suggest that YC-1 enhanced the learning behavior during the acquired session and also the long-term memory.

\subsection{Involvement of NO-cGMP-PKG and ERK pathway in the memory} enhancement by $\mathrm{YC}-1$

Since NO-cGMP-PKG pathway is involved in the learning and memory potentiating action of YC-1 in water maze test (Chien et al., 2005), we then further investigated the effect of L-NAME, PD98059 as well as Rp-8-Br-PET-cGMP on the enhancement of learning and memory by YC-1 in shuttle avoidance test. In these experiments, rats chronically implanted with a cannulae were slowly injected (intracerebroventrically, $1 \mu \mathrm{L} / \mathrm{min})$ with NOS inhibitor L-NAME (1 $\mu \mathrm{mol})$, PKG inhibitor Rp-8-Br-PET-cGMPS (1 nmol) or MEK inhibitor PD98059 $(1 \mathrm{nmol})$ concomitantly with i.p. injection of YC-1 (1 mg/kg) $10 \mathrm{~min}$ before each daily training. As shown in Fig. 2C, L-NAME, Rp-8-Br-PETcGMPS as well as PD98059 markedly antagonized the enhancing effect of YC-1 on learning and memory (ANOVA test among two groups: $\left.F_{3,38}=62.575, P<0.001\right)$. These results suggest that the potentiation of fear memory by YC- 1 is sensitive to the inhibitors of NO/PKG/MEK signaling pathway.

\subsection{Increase of phosphorylation of ERK and Akt by YC-1}

We further examined whether YC-1 affects MAPK activation in response to active shuttle avoidance test. It has been reported that the activation of ERK and Akt reaches peak between $30 \mathrm{~min}$ and $1 \mathrm{~h}$ postfear conditioning (Schafe et al., 2000; Lin et al., 2001). Thirty minutes after behavior tests on Day-1, rats were sacrificed, and the tissues of amygdala, hippocampus, cortex and cerebellum were removed separately for analysis. The results show that there was a significant increase in p-ERK and p-Akt in amygdala but not in hippocampus or cortex 30 min after training test on Day-1 (Fig. 3, A and B). YC-1 significantly increased the activation of ERK and Akt, indicating that the enhancement of learning and memory by YC-1 may result from the potentiation of ERK and Akt signaling pathways. Two hours after training on Day-1, both ERK and Akt were still highly activated in YC1-treated group (Fig. 3, C and D).
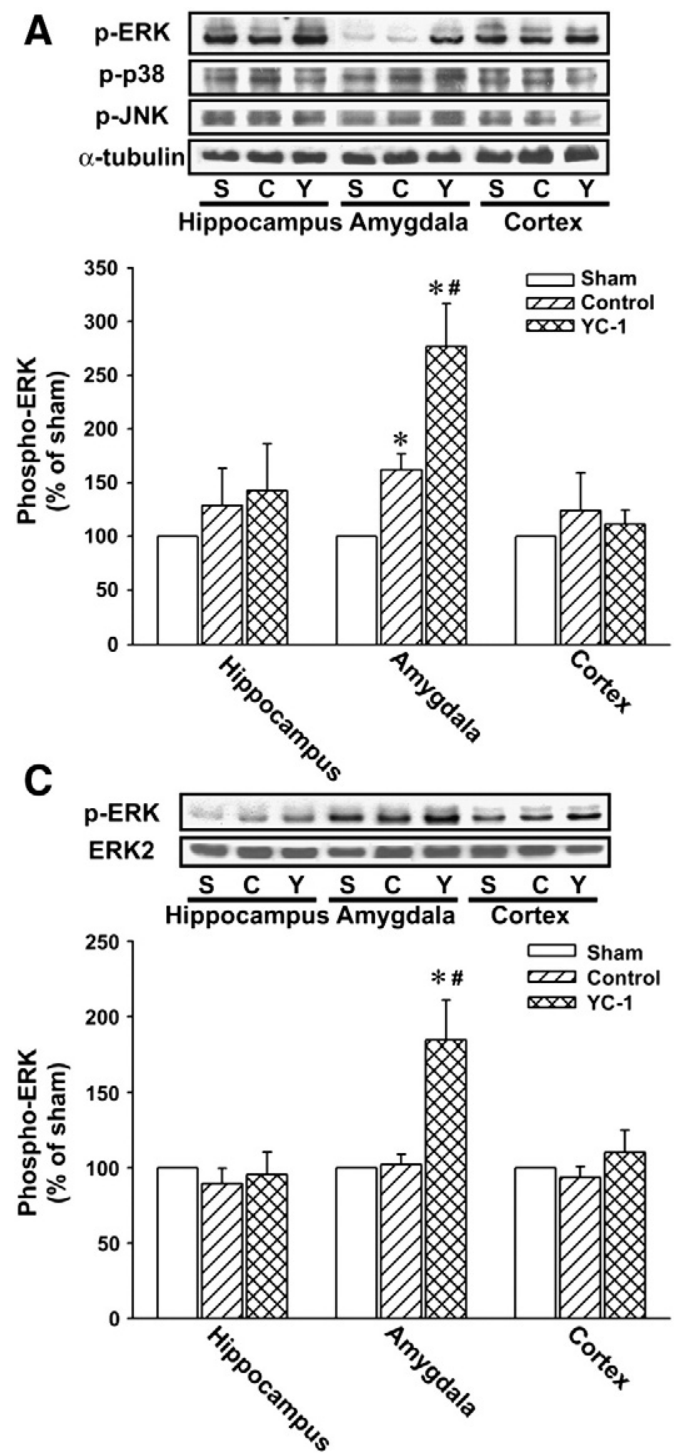

B
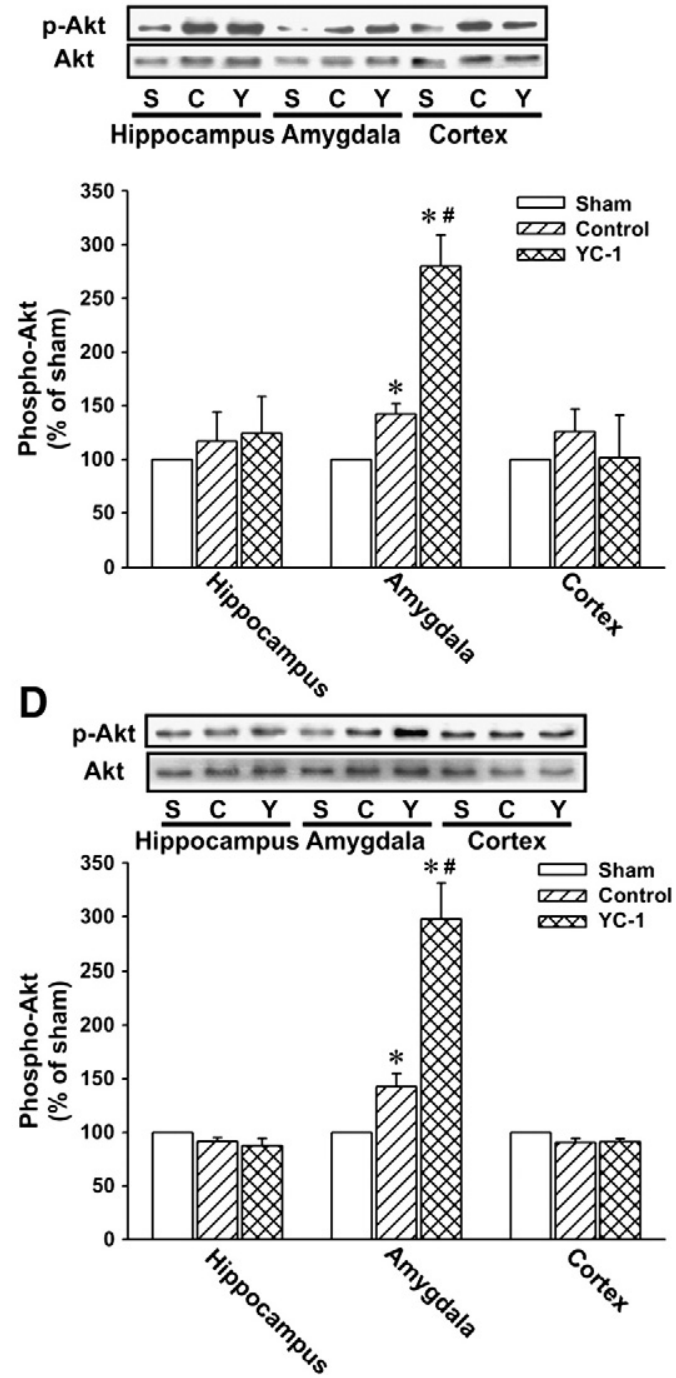

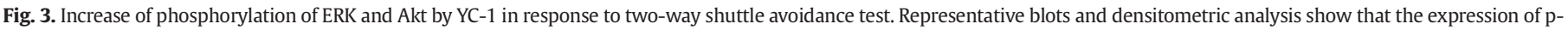

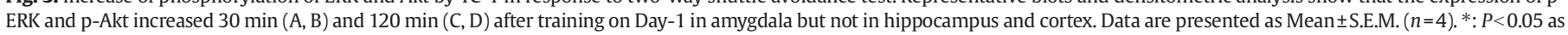
compared with sham-group. \#: $P<0.05$ as compared with control-group. 


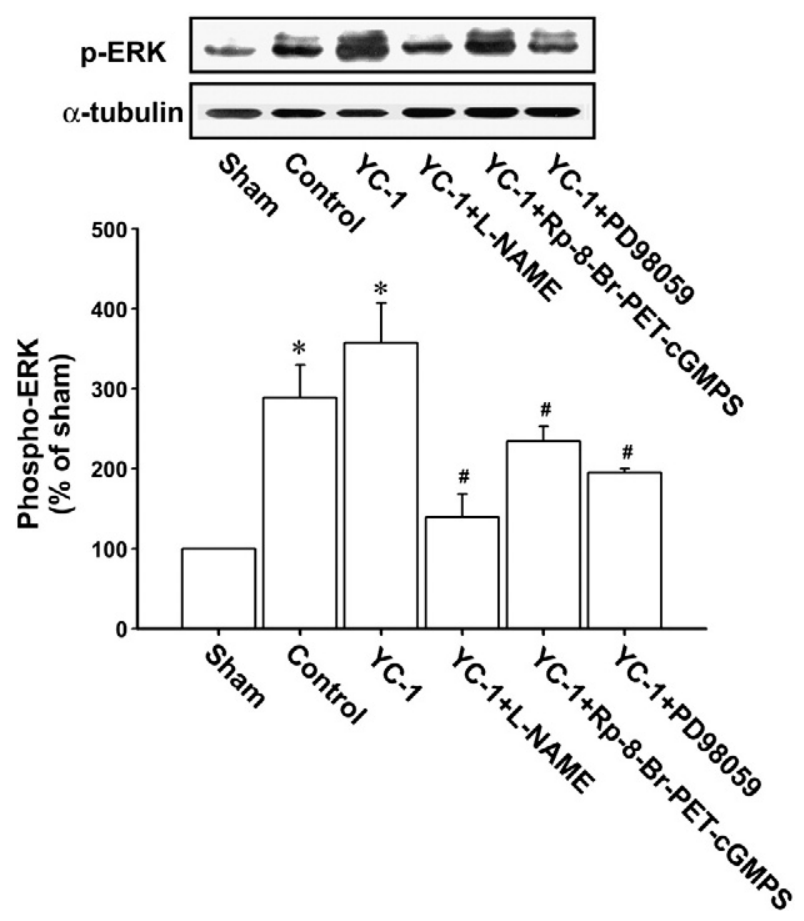

Fig. 4. YC-1-induced increase of ERK phosphorylation is antagonized by L-NAME, Rp8-Br-PET-cGMP and PD98059. Representative blots and densitometric analysis show that the expression of p-ERK in amygdala increased 30 min after training in two-way active shuttle avoidance test on Day-4. YC-1 $(1 \mathrm{mg} / \mathrm{kg} / \mathrm{day}$, i.p.)-induced potentiation of ERK phosphorylation was antagonized by L-NAME, Rp-8-Br-PET-cGMP, and PD98059 (i.c.v., doses were the same as those in Fig. 2). Data are presented as Mean \pm S.E.M. $(n=3)$. ${ }^{*}: P<0.05$ as compared with sham-group. \#: $P<0.05$ as compared with YC-1treated group.

To confirm that enhancement of fear memory formation by YC-1 was through the increase of the phosphorylation of ERK and Akt, YC-1 was injected intraperitoneally (1 $\mathrm{mg} / \mathrm{kg}$ ) and enzyme inhibitors were administered intracerebroventrically 10 min prior to training on each day. Immunoblotting for p-ERK in amygdala was performed $30 \mathrm{~min}$ after training on Day-4. As shown in Fig. 4, L-NAME, Rp-8-Br-PETcGMPS and PD98059 significantly antagonized the potentiation of ERK phosphorylation by YC-1. These results indicate that NO/PKG/MEK signaling pathways are probably involved in memory enhancement by YC-1 in active shuttle avoidance test.

3.5. Increase of c-Fos expression by $Y C-1$ in response to active shuttle avoidance test

Previous reports indicate that exposure to a conditioned stimulusunconditioned stimulus paired fear conditioning elevated c-Fos protein expression in amygdala $2 \mathrm{~h}$ after shuttle avoidance test (Savonenko et al., 2003). Our results are consistent with this speculation and it was found that YC-1-treated rats obtained a higher level of c-Fos expression in both amygdala and cortex $2 \mathrm{~h}$ after training on Day- 1 and a better response rate in the following training days. Both immunoblotting and immunohistochemistry showed an increase of c-Fos in amygdala of YC-1-treated rats (Fig. 5).

3.6. Increase of $B D N F$ expression by $Y C-1$ in response to active shuttle avoidance test

There is a good correlation between BDNF mRNA expression and behavioral performance in various kinds of learning and memory tests (Mizuno et al., 2000; Tyler et al., 2002), possibly through CREBdependent mechanisms (Alonso et al., 2005; Monti et al., 2005). We therefore further investigated the effect of YC-1 on the expression of neurotrophin BDNF. The amygdala was isolated for mRNA analysis

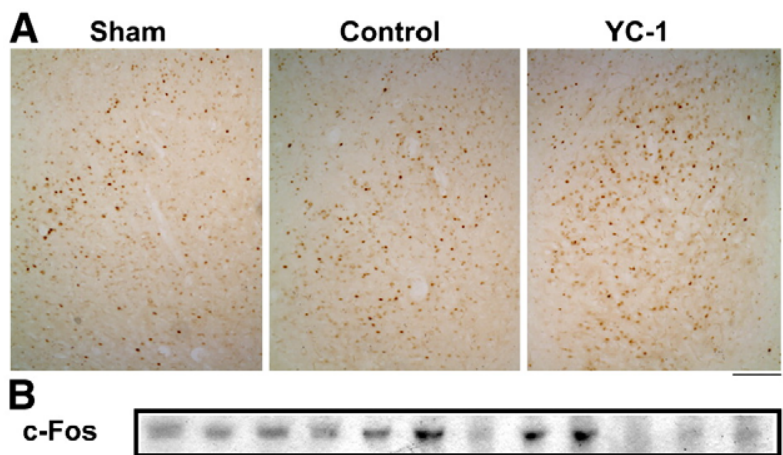

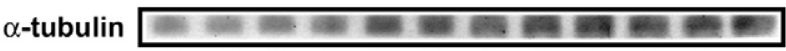
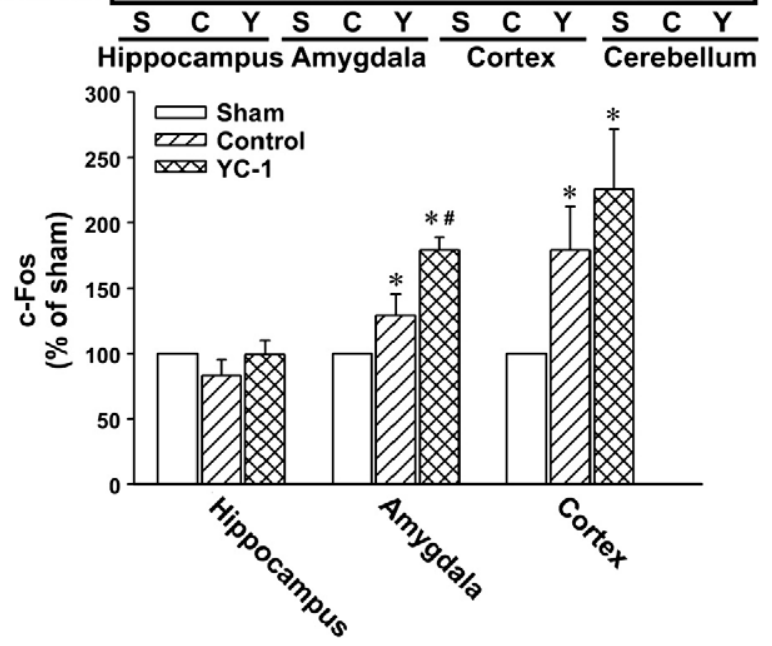

Fig. 5. Increase of c-Fos expression by YC-1 in response to two-way active shuttle avoidance test. (A) Increase of immunostaining of c-Fos by YC-130 min after training on Day-2. Scale bar, $1 \mathrm{~mm}$. (B) Representative blots and densitometric analysis show that the expression of c-Fos in amygdala was enhanced by YC-1. Data are presented as Mean \pm S.E.M. $(n=4)$. *: $P<0.05$ as compared with sham-group. \#: $P<0.05$ as compared with control group.

30 min after avoidance training. There was no change of BDNF expression on Day-1. However, it was found that the learning behavior increased the mRNA expression of BDNF and CREB on Day-2 and YC-1
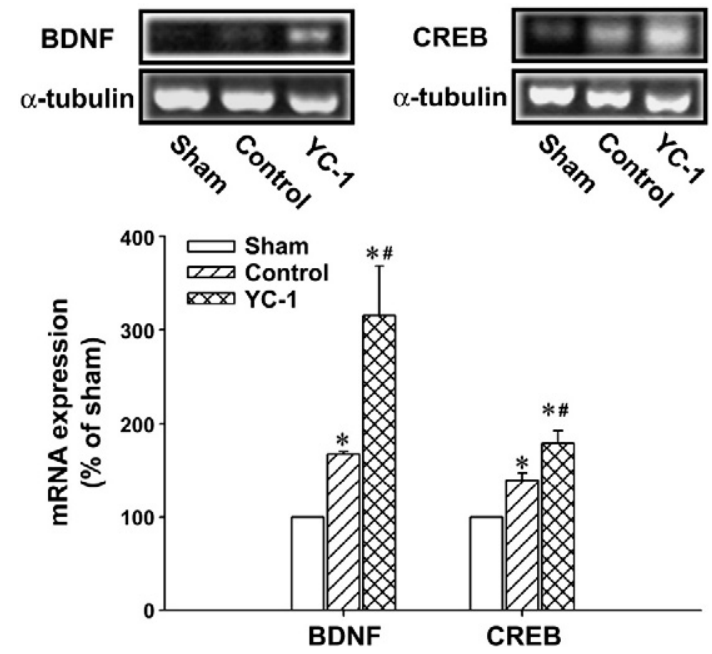

Fig. 6. Increase of BDNF and CREB mRNA by YC-1 in response to two-way active shuttle avoidance test. Representative blots and densitometric analysis show that the expression of BDNF and CREB mRNA in amygdala increased $30 \mathrm{~min}$ after training in two-way active shuttle avoidance test on Day-2 and YC-1 enhanced the responses. Data are presented as Mean \pm S.E.M. $(n=3)$. *: $P<0.05$ as compared with sham-group. \#: $P<0.05$ as compared with control group. 

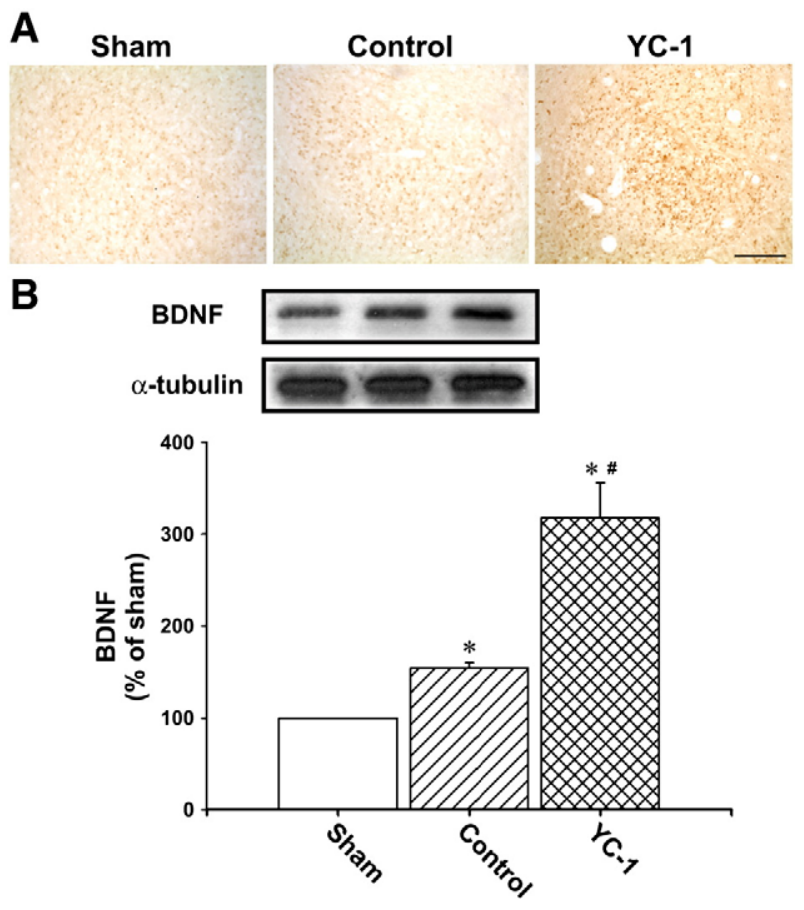

Fig. 7. Upregulation of BDNF protein by $\mathrm{YC}-1$ in response to two-way active shuttle avoidance test. (A) Increase of immunostaining of BDNF by YC-1 30 min after training on Day-2. Note that compared with control, treatment with YC-1 markedly increased the BDNF immunoreactivity-positive cells in amygdala. Scale bar, $1 \mathrm{~mm}$. (B) Representative blots and densitometric analysis show that the expression of BDNF in amygdala increased $30 \mathrm{~min}$ after training in two-way active shuttle avoidance test on Day-2, and YC-1 potentiated the response. Data are presented as Mean \pm S.E.M. $(n=3)$. ${ }^{*}: P<0.05$ as compared with sham-group. \#: $P<0.05$ as compared with control group.

treatment potentiated this phenomenon (Fig. 6). We further examined the immunostaining and protein level of BDNF in amygdala $2 \mathrm{~h}$ after training on Day-2. Both immunoblotting and immunohistochemistry showed that YC-1 increased the protein expression of BDNF in amygdala (Fig. 7).

\section{Discussion}

In the present study, we examined the effect of YC-1 on the performance of active avoidance learning tasks. It was demonstrated that the avoidance response in YC-1-treated rats was significantly higher as compared to controls. The present findings are in agreement with our previous studies, which demonstrate the positive effect of YC-1 on Morris water maze and passive avoidance test. The beneficial effects of YC-1 on learning behavior appear to be mainly associated with the NO-cGMP-PKG pathway. We here further examined the effect of YC-1 on the expression of BDNF and c-Fos related to learning behavior.

The active shuttle avoidance task is considered for measuring nondeclarative memory and relies on both classical fear conditioning and instrumental conditioning (Savonenko et al., 2003). Two types of classical conditioning can be considered, the delay and the trace paradigm. In the delay fear conditioning, the conditioned stimulus is overlapped and coterminated with an unconditioned stimulus or immediately followed by an unconditioned stimulus. In trace conditioning, a time gap is introduced between the end of the conditioned stimulus and the start of the unconditioned stimulus. The delay conditioning is the usual practice for conditioning (Carageorgiou et al., 2003; Torras-Garcia et al., 2003; Lopez-Aumatell et al., 2008; Tsoory et al., 2008). It has been reported that hippocampus and anterior cingulate cortex are associated with trace but not the delay fear conditioning, indicating that these two procedures are dependent on different brain structures (Han et al., 2003; Shors, 2004).
When pursuing the active avoidance task, differences could be caused by visual sensitivity, shock reactivity, locomotor activity, and levels of stress hormones, rather than learning, per se. Our previous study has shown that there is no change in the visual sensitivity or shock reactivity in YC-1-treated rats (Chien et al., 2005). The frequency of door crossing during the exploration time was not affected by YC-1 treatment, indicating the exploring activity was the same between two groups (Fig. 2A). In addition, there was no difference in locomotor activity between control and YC-1-treated group, indicating that the effect on active avoidance test was not due to exercise inability (data not shown).

Nitric oxide is produced postsynaptically and retrogradely diffuses to act on the presynaptic cells, which in turn modulates the release of neurotransmmiters (Hawkins et al., 1998). Some studies have indicated that NO may act at two different sites (pre- and postsynaptic) to contribute synaptic plasticity (Lu and Hawkins, 2002). It is also reported that the ERK activation is required for the acquisition, consolidation and extinction of fear memory (Adams and Sweatt, 2002; Shalin et al., 2004), but only a few papers discuss the relationship of ERK and PKG (Komalavilas et al., 1999; Yamazaki et al., 2005). NO itself has the ability to induce neurite outgrowth and that NO-induced ERK activation may act through the NO-cGMP-PKG signaling pathway (Yamazaki et al., 2005).

Recently, the important role of PI-3 K and Akt in the fear memory has also been explored (Ou and Gean, 2006). Inhibition of PI-3 K and Akt activation in amygdala antagonized the synaptic plasticity and memory consolidation, and PI-3K activation may occur at a point upstream of MAPK (Lin et al., 2001). To investigate the signaling mechanism of YC-1, we here further confirm that the potentiating effect of YC-1 on fear memory was mainly through NO/cGMP/PKG/ERK signaling pathway. It was found that YC-1 markedly increased the activation of ERK and Akt in amygdala after fear conditioning (Fig. 3). The delay fear conditioning is thought to be related to the amygdala but not to the hippocampus or cortex (Han et al., 2003; Shors, 2004). Therefore, the activation of ERK and Akt appeared in amygdala but not in hippocampus or cortex. The activation of c-Fos was mainly in amygdala and cortex, but not in hippocampus or cerebellum (Fig. 5).

As shown in Fig. 3, there is an increase in ERK and Akt phosphorylation in controls as compared to shams. This result is consistent with previous reports that activation of ERK and Akt in the lateral amygdala is specific to associative conditioned stimulusunconditioned stimulus pairing (Schafe et al., 2000; Lin et al., 2001). Non-associative factors such as auditory stimulation alone or foot shock alone did not induce the activation of ERK (Schafe et al., 2000). The increasing expression of c-Fos, CREB and BDNF in control group as compared to sham was related to associative pairing of conditioned stimulus and unconditioned stimulus rather than reflecting nonassociative processes. When the rats received 4 days' training, the control rats also established fear memory. The activation of ERK was not statistically different between control and YC-1-treated rats, but it was significantly inhibited by L-NAME, Rp-8-Br-PET-cGMPS and PD98059.

The longer activation of ERK and Akt was induced by YC-1, suggesting that the signaling pathway of YC-1 could interact with protein phosphatase. It was reported that the G-substrate, a specific substrate of NO-cGMP-PKG signaling pathway, acts as an endogenous protein phosphatase inhibitor (Nakazawa et al., 2005). Further investigation is needed regarding whether YC-1 enhances the activation period of ERK and Akt through the inhibition of protein phosphatase or not. CREB is essential for maintenance of longer lasting components of LTP and is believed to be required for formation of new memories (Finkbeiner et al., 1997; Josselyn et al., 2001). It is also reported that NO-cGMP-PKG signaling pathway contributes to CREB phosphorylation and LTP in hippocampus by causing or amplifying intracellular $\mathrm{Ca}^{2+}$ release (Lu et al., 1999; Lu and Hawkins, 2002). Here we found that YC-1 increased CREB expression in amygdala in 
response to shuttle avoidance test. It was also demonstrated that BDNF expression increased in amygdala in response to shuttle avoidance test. The ability of YC- 1 in enhancing learning and memory may result from an increase of BDNF expression in amygdala. The amygdala involved in Pavlovian fear conditioning provides us new insights on the mechanisms of BDNF-mediated synaptic plasticity during the learning and memory process (Rattiner et al., 2005).

The remarkable characteristics of YC- 1 in potentiating NOstimulated guanylate cyclase activity during the induction of activity-dependent synaptic plasticity and the high spatial and temporal specificity of NO-induced cellular actions may explain why YC-1 enhances the acquisition of new memory. Our results suggest that YC1 may be a good candidate for improving learning and memory in humans. These affirmative changes may be related to the increase of BDNF expression. Further studies are required to clarify the relationship between NO signaling and BDNF expression.

\section{Acknowledgement}

This work was supported by research grants from the National Science Council, Taiwan.

\section{References}

Adams, J.P., Sweatt, J.D., 2002. Molecular psychology: roles for the ERK MAP kinase cascade in memory. Annu. Rev. Pharmacol. Toxicol. 42, 135-163.

Alonso, M., Bekinschtein, P., Cammarota, M., Vianna, M.R., Izquierdo, I., Medina, J.H. 2005. Endogenous BDNF is required for long-term memory formation in the rat parietal cortex. Learn Mem. 12, 504-510.

Bailey, C.H., Kandel, E.R., Si, K., 2004. The persistence of long-term memory: a molecular approach to self-sustaining changes in learning-induced synaptic growth. Neuron $44,49-57$

Carageorgiou, H., Zarros, A., Tsakiris, S., 2003. Selegiline long-term effects on brain acetylcholinesterase, $(\mathrm{Na}+, \mathrm{K}+)$-ATPase activities, antioxidant status and learning performance of aged rats. Pharmacol. Res. 48, 245-251.

Chen, X., Garelick, M.G., Wang, H., Lil, V., Athos, J., Storm, D.R., 2005. PI3 kinase signaling is required for retrieval and extinction of contextual memory. Nat. Neurosci. 8, 925-931.

Chhatwal, J.P., Stanek-Rattiner, L., Davis, M., Ressler, K.J., 2006. Amygdala BDNF signaling is required for consolidation but not encoding of extinction. Nat. Neurosci. 9, $870-872$.

Chien, W.L., Liang, K.C., Teng, C.M., Kuo, S.C., Lee, F.Y., Fu, W.M., 2003. Enhancement of long-term potentiation by a potent nitric oxide-guanylyl cyclase activator, 3-(5hydroxymethyl-2-furyl)-1-benzyl-indazole. Mol. Pharmacol. 63, 1322-1328.

Chien, W.L., Liang, K.C., Teng, C.M., Kuo, S.C., Lee, F.Y., Fu, W.M., 2005. Enhancement of learning behaviour by a potent nitric oxide-guanylate cyclase activator YC-1. Eur. J. Neurosci. 21, 1679-1688.

Finkbeiner, S., Tavazoie, S.F., Maloratsky, A., Jacobs, K.M., Harris, K.M., Greenberg, M.E., 1997. CREB: a major mediator of neuronal neurotrophin responses. Neuron 19 1031-1047.

Francia, N., Cirulli, F., Chiarotti, F., Antonelli, A., Aloe, L., Alleva, E., 2006. Spatial memory deficits in middle-aged mice correlate with lower exploratory activity and a subordinate status: role of hippocampal neurotrophins. Eur. J. Neurosci. 23, 711-728.

Han, C.J., O'Tuathaigh, C.M., van Trigt, L., Quinn, J.J., Fanselow, M.S., Mongeau, R., Koch, C., Anderson, D.J., 2003. Trace but not delay fear conditioning requires attention and the anterior cingulate cortex. Proc. Natl. Acad. Sci. U. S. A. 100, 13087-13092.

Hawkins, R.D., Son, H., Arancio, O., 1998. Nitric oxide as a retrograde messenger during long-term potentiation in hippocampus. Prog. Brain Res. 118, 155-172.

Herry, C., Trifilieff, P., Micheau, J., Luthi, A., Mons, N., 2006. Extinction of auditory fear conditioning requires MAPK/ERK activation in the basolateral amygdala. Eur. J. Neurosci. 24, 261-269.

Johnson, D.M., Baker, J.D., Azorlosa, J.L., 2000. Acquisition, extinction, and reinstatement of Pavlovian fear conditioning: the roles of the NMDA receptor and nitric oxide. Brain Res. 857, 66-70.

Josselyn, S.A., Shi, C., Carlezon, W.A., Jr Neve, R.L., Nestler, E.J., Davis, M., 2001. Long-term memory is facilitated by cAMP response element-binding protein overexpression in the amygdala. J. Neurosci. 21, 2404-2412.

Kim, J.J., Jung, M.W., 2006. Neural circuits and mechanisms involved in Pavlovian fear conditioning: a critical review. Neurosci. Biobehav. Rev. 30, 188-202.

Komalavilas, P., Shah, P.K., Jo, H., Lincoln, T.M., 1999. Activation of mitogen-activated protein kinase pathways by cyclic GMP and cyclic GMP-dependent protein kinase in contractile vascular smooth muscle cells. J. Biol. Chem. 274, 34301-34309.

LeDoux, J.E., 2000. Emotion circuits in the brain. Annu. Rev., Neurosci. 23, 155-184.
Lin, C.H., Yeh, S.H., Lin, C.H., Lu, K.T., Leu, T.H., Chang, W.C., Gean, P.W., 2001. A role for the PI-3 kinase signaling pathway in fear conditioning and synaptic plasticity in the amygdala. Neuron 31, 841-851.

Lopez-Aumatell, R., Guitart-Masip, M., Vicens-Costa, E., Gimenez-Llort, L., Valdar, W. Johannesson, M., Flint, J., Tobeña, A., Fernandez-Teruel, A., 2008. Fearfulness in a large $\mathrm{N} / \mathrm{Nih}$ genetically heterogeneous rat stock: differential profiles of timidity and defensive flight in males and females. Behav. Brain Res. 188, 41-55.

Lu, Y.F., Hawkins, R.D., 2002. Ryanodine receptors contribute to cGMP-induced latephase LTP and CREB phosphorylation in the hippocampus. J. Neurophysiol. 88, 1270-1278.

Lu, Y.F., Kandel, E.R., Hawkins, R.D., 1999. Nitric oxide signaling contributes to late-phase LTP and CREB phosphorylation in the hippocampus. J. Neurosci. 19, 10250-10261.

Maren, S., 2005. Synaptic mechanisms of associative memory in the amygdala. Neuron 47, 783-786.

Mizuno, M., Yamada, K., Olariu, A., Nawa, H., Nabeshima, T., 2000. Involvement of brainderived neurotrophic factor in spatial memory formation and maintenance in a radial arm maze test in rats. J. Neurosci. 20, 7116-7121.

Monti, B., Berteotti, C., Contestabile, A., 2005. Dysregulation of memory-related proteins in the hippocampus of aged rats and their relation with cognitive impairment. Hippocampus 15, 1041-1049.

Nakazawa, T., Endo, S., Shimura, M., Kondo, M., Ueno, S., Tamai, M., 2005. Retinal Gsubstrate, potential downstream component of NO/cGMP/PKG pathway, is located in subtype of retinal ganglion cells and amacrine cells with protein phosphatases. Brain Res. Mol. Brain Res. 135, 58-68.

Opazo, P., Watabe, A.M., Grant, S.G., O'dell, T.J., 2003. Phosphatidylinositol 3-kinase regulates the induction of long-term potentiation through extracellular signalrelated kinase-independent mechanisms. J. Neurosci. 23, 3679-3688.

Ou, L.C., Gean, P.W., 2006. Regulation of amygdala-dependent learning by brain-derived neurotrophic factor is mediated by extracellular signal-regulated kinase and phosphatidylinositol-3-kinase. Neuropsychopharmacology 31, 287-296.

Ou, L.C., Gean, P.W., 2007. Transcriptional regulation of brain-derived neurotrophic factor in the amygdala during consolidation of fear memory. Mol. Pharmacol. 72, 350-358.

Paxinos, G., Watson, G., 1997. The Rat Brain in Sterotoxic Coordinates. Academic Press San Diego.

Poo, M.M., 2001. Neurotrophins as synaptic modulators. Nat. Rev., Neurosci. 2, 24-32.

Prast, H., Philippu, A., 2001. Nitric oxide as modulator of neuronal function. Prog. Neurobiol. 64, 51-68.

Rattiner, L.M., Davis, M., Ressler, K.J., 2005. Brain-derived neurotrophic factor in amygdala-dependent learning. Neuroscientist 11, 323-333.

Rodrigues, S.M., Schafe, G.E., LeDoux, J.E., 2004. Molecular mechanisms underlying emotional learning and memory in the lateral amygdala. Neuron 44, 75-91.

Sato, T., Suzuki, E., Yokoyama, M., Watanabe, S., Miyaoka, H., 2006. Auditory fear conditioning and conditioned stress raise $\mathrm{NO}_{3}^{-}$level in the Amygdala. Neuropsychobiology 53, 142-147.

Savonenko, A., Werka, T., Nikolaev, E., Zieliñski, K., Kaczmarek, L., 2003. Complex effects of NMDA receptor antagonist APV in the basolateral amygdala on acquisition of two-way avoidance reaction and long-term fear memory. Learn. Mem. 10, 293-303.

Schafe, G.E., Atkins, C.M., Swank, M.W., Bauer, E.P., Sweatt, J.D., LeDoux, J.E., 2000 Activation of ERK/MAP kinase in the amygdala is required for memory consolidation of Pavlovian fear conditioning. J. Neurosci. 20, 8177-8187.

Schafe, G.E., Bauer, E.P., Rosis, S., Farb, C.R., Rodrigues, S.M., LeDoux, J.E., 2005a. Memory consolidation of Pavlovian fear conditioning requires nitric oxide signaling in the lateral amygdala. Eur. J. Neurosci. 22, 201-211.

Schafe, G.E., Doyere, V., LeDoux, J.E., 2005b. Tracking the fear engram: the latera amygdala is an essential locus of fear memory storage. J. Neurosci. 25, 10010-10014

Shalin, S.C., Zirrgiebel, U., Honsa, K.J., Julien, J.P., Miller, F.D., Kaplan, D.R., Sweatt, J.D. 2004. Neuronal MEK is important for normal fear conditioning in mice. J. Neurosci. Res. 75, 760-770.

Shors, T.J., 2004. Memory traces of trace memories: neurogenesis, synaptogenesis and awareness. Trends Neurosci. 27, 250-256.

Soule, J., Messaoudi, E., Bramham, C.R., 2006. Brain-derived neurotrophic factor and contro of synaptic consolidation in the adult brain. Biochem. Soc. Trans. 34, 600-604.

Sweatt, J.D., 2004. Mitogen-activated protein kinases in synaptic plasticity and memory. Curr. Opin. Neurobiol. 14, 311-317.

Torras-Garcia, M., Costa-Miserachs, D., Morgado-Bernal, I., Portell-Cortes, I., 2003. Improvement of shuttle-box performance by anterodorsal medial lessions in rats. Behav. Brain Res. 141, 147-158.

Tsoory, M., Guterman, A., Richter-Levin, G., 2008. Exposure to stressors during juvenility disrupts development-related alterations in the PSA-NCAM to NCAM expression ratio: potential relevance for mood and anxiety disorders. Neuropsychopharmacology 33, 378-393.

Tyler, W.J., Alonso, M., Bramham, C.R., Pozzo-Miller, L.D., 2002. From acquisition to consolidation: on the role of brain-derived neurotrophic factor signaling in hippocampal-dependent learning. Learn Mem. 9, 224-237.

Wang, H., Hu, Y., Tsien, J.Z., 2006. Molecular and systems mechanisms of memory consolidation and storage. Prog. Neurobiol. 79, 123-135.

Yamazaki, M., Chiba, K., Mohri, T., 2005. Fundamental role of nitric oxide in neuritogenesis of PC12h cells. Br. J. Pharmacol. 146, 662-669. 